Background The Hospital Infections and Antibiotic Policy Committee guidelines recommend antibiotics to cover coagulase-negative staphylococcus and Gram-negative bacilli with vancomycin + aminoglycoside or aztreonam if Catheter-Related Bacteraemia (CRB) is suspected. Fungal coverage has to be evaluated.

Purpose To assess compliance with the antibiotic treatment protocol in the CRB in the Intensive Care Unit (ICU).

Materials and Methods Observational prospective 6-month study in a 32-bed ICU in a tertiary hospital in patients hospitalised $\geq 48$ hours carrying a Central Venous Catheter (CVC).

Demographic and antibiotic treatment were recorded and compared with the empirical treatment recommended.

Results From 8 September 2011 to 8 March 2012, 596 patients were admitted to ICU; 571 patients used CVC; 390 (68.3\%) males, mean age $61.0 \pm 15.6$ years; the number of CVC used was 844 , equivalent to 5578 CVC days

During this period 114 CVCs were removed in patients with fever and 11 cases of CRB were confirmed (10 patients); incidence $1.97 \mathrm{CRB} / 1000$ CVC days.

Microbiology: 1 Morganella morganii (treatment levofloxacin + piperacillin/tazobactam); 2 methicillin-sensitive Staphylococcus aureus (one treated with meropenem, another levofloxacin + teicoplanin); 3 Staphylococcus epidermidis (one treated with linezolid, the second with piperacillin/tazobactam + teicoplanin, and the last with linezolid + meropenem + caspofungin); 1 Escherichia coli (treatment piperacillin/tazobactam); 1 Pseudomonas aeruginosa (treatment piperacillin/tazobactam); 2 carbapenemase-positive Klebsiella pneumoniae (treated with piperacillin/tazobactam + voriconazole) and 1 Candida glabrata (patient received fluconazole + levofloxacin).

Empiric antibiotic treatment wasn't correct in 8 cases of CRB, lacking empirical Gram-positive coverage in 7 cases and Gramnegative in 1 case. However, according to microbiological results, bacteraemia coverage was correct in $90 \%$.

Conclusions Protocol compliance is low in the ICU for empirical treatment of CRB. A large number of CVCs were removed for fever with no clear correlation with CRB. Patients with fever of unknown origin receive broad-spectrum antibiotic treatment including antibiotic coverage of a wider spectrum than is strictly necessary for CVC infection. Yet $72.72 \%$ of patients would not receive appropriate empirical treatment if CRB was suspected.

No conflict of interest.

\section{GRP-038 CHEMOTHERAPY-INDUCED NAUSEA AND VOMITING IN BREAST CANCER PATIENTS: EFFECTIVENESS AND SAFETY OF ANTIEMETIC TREATMENT}

doi:10.1136/ejhpharm-2013-000276.038

'E Domingo Chiva, 'MJ De Mora Alfaro, 'E García Martínez, 'MR Garrigues Sebastiá, 'C García Gómez, 'M Hernandez Sansalvador, ${ }^{2 B}$ Medrano Martínez. ${ }^{1}$ Complejo Hospitalario Universitario de Albacete, Servicio de Farmacia, Albacete, Spain; ${ }^{2}$ Complejo Hospitalario Universitario de Albacete, Hospital de Día de Oncología, Albacete, Spain

Background Chemotherapy-induced nausea and vomiting are two of the most frequent manifestations that appear in cancer patients that significantly affect the course of their disease.

Purpose The objectives of this study are:

- to describe the antiemetic treatment used in patients with breast cancer treated with chemotherapy,

- to determine the degree of adaptation to the good clinical practise guides for the management of this type of complication, in other words how closely treatment followed the ASCO, MASCC and NCCN guideline recommendations,

- to analyse the effectiveness of those treatments and known adverse reactions that patients may suffer because of antiemetic or chemotherapy drugs.
Materials and Methods A descriptive, transversal and observational study of one month. The study included breast cancer patients from the day hospital who had received at least one previous chemotherapy cycle. Variables were collected using a questionnaire completed by the patient and pharmacy service software.

Results Of 47 patients, 32 agreed to participate in the study, with a mean age of 50.7 years $(\mathrm{SD}=9.8)$. On day 1 post-chemotherapy, $34.4 \%$ of treatments did not follow the guidelines and on days 2,3 and 4 this increased to $46.9 \% .31 .3 \%$ of patients experienced acute nausea and $15.6 \%$ acute emesis, $43.8 \%$ developed late nausea and $18.75 \%$ late vomiting. The number of patients with anticipatory nausea and vomiting was lower. The complete response to antiemetic treatment (absence of nausea, vomiting and need for antiemetic rescue medication) was achieved in $50 \%$ of patients. The most common averse events suffered by patients were fatigue, weakness (75.0\%) and insomnia (56.3\%).

Conclusions The lack of compliance with guidelines together with the results obtained of inefficiency of the treatment mean that we require new therapeutic strategies to allow us to obtain better control of emesis.

No conflict of interest.

\section{GRP-039 CLASSIFICATION OF THE PHARMACEUTICAL INTERVENTIONS MADE USING THE ISOFAR PROGRAMME}

doi:10.1136/ejhpharm-2013-000276.039

J Diaz-Navarro, JF Lopez-Vallejo, E Rios-Sanchez, R Castaño-Lara, S Fenix-Caballero, JM Borrero-Rubio, EJ Alegre-del-Rey. Hospital Universitario de Puerto Real, pharmacy, Puerto Real (Cádiz), Spain

Background A variety of errors in the medication process means reduced safety for the patient and less effective treatment.

Purpose To analyse from the Unidosis area the types of intervention, medicines-related problems (MRPs), impact and savings recorded in the ISOFAR programme.

Materials and Methods A retrospective analysis was performed of the interventions made by the Pharmacy service since the establishment of the ISOFAR programme (from March 2007 to April 2011). Each intervention was recorded and a note made in the patient data: type of intervention, MRPs, impact and savings of the intervention.

Results In the period of the study a total of 6116 interventions covering: change of drug (52\%), maintenance of treatments not included in the Hospital Pharmacotherapeutic Guide (23\%), incomplete medical orders (15\%), discontinued drugs (4\%) and other reasons $(6 \%)$ were recorded. The MRPs detected with the interventions were classified as: change by Therapeutic Exchange Protocol (TEP) (26.8\%), necessary drug but not included in the TEP $(22.9 \%)$, no adjustment to protocols (14.6\%), change discussed (10.1\%) and incomplete order $(2.1 \%)$. In $53 \%$ the impact of the intervention was on effectiveness and in $24 \%$ on safety. The total savings in the evaluated period reached $184,153.47$ euros.

Conclusions The most frequent intervention was a change of medicine probably due to the physician's ignorance of the Hospital Pharmacotherapeutic Guide and the Therapeutic Exchange Proto$\mathrm{col}$; therefore it would be appropriate to consider the inclusion of new drugs in the HPG. A high percentage of medical orders were badly written, so the patient did not receive the medicine. The interventions were intended to improve the efficacy and safety of the prescribed drugs and moreover provide an important financial saving.

No conflict of interest. 